\title{
Percutaneous Nephrolithotomy for Staghorn Stones in Patients with Solitary Kidney in Prone Position or in completely Supine Position: a Single-center Experience
}

\author{
Yanbo Wang, Yuchuan Hou, Fengming Jiang, Yan Wang, Chunxi Wang \\ Department of Urology, First Hospital of Jilin University, Changchun, China
}

\section{ABSTRACT}

Purpose: To evaluate the effectivity and safety of percutaneous nephrolithotomy (PCNL) in the treatment of solitary kidney with staghorn stones in prone position or in completely supine position.

Materials and methods: We retrospectively reviewed the records of 18 patients with staghorn stones in a solitary kidney treated with PCNL. 12 patients underwent PCNL in prone position (group A). 6 patients underwent PCNL in completely supine position (group B). Demographic data, number of accesses, operating time, stone free rate, hemoglobin values, hospital stay and complications were studied. Serum creatinine, systolic and diastolic blood pressure, and new onset hypertension were determined preoperatively and postoperatively at 3 months.

Results: No blood transfusions were required and no abdominal or thoracic organ injuries were reported in both groups. The mean operative time was 104 minutes (range:72-145 minutes) and 128 minutes (range:80-170 minutes), respectively. The I stage stone free rate was $91.7 \%$ and $83.3 \%$, respectively. There was no new onset hypertension by the end of follow-up in both groups. Both groups showed a similar fall in serum creatinine at 3 month follow-up period ( $p=0.004$ and 0.029 , respectively). Systolic blood pressure showed a statistically significant improvement in group B ( $p=0.034)$. Conclusion: PCNL is safe and has an acceptably high stone free rate in patients with solitary kidneys in both prone and completely supine position. At short-term follow-up, systolic blood pressure had improved in PCNL in supine position.

\section{ARTICLE INFO}

\section{Key words:}

Nephrostomy, Percutaneous;

Kidney; Urinary Bladder

Calculi; Prone Position; Supine

Position

Int Braz J Urol. 2012; 38: 788-94

Submitted for publication:

May 16, 2012

Accepted after revision:

August 01, 2012

\section{INTRODUCTION}

Staghorn calculi are branched stones that occupy a large portion of the collecting system, typically flling the renal pelvis and branching into several or all of the calices (1). Treatment of staghorn stones in patients with solitary kidney is one of the most challenging problems in urology (2). In this study, we evaluated the safety and efficacy of PCNL in the treatment of staghorn stones both in prone position and in completely supine position in a solitary kidney. To the best of our knowledge, this study is the first series reported in the literature.

\section{MATERIALS AND METHODS}

Clinical data

We retrospectively reviewed the records of 18 patients with solitary kidneys who had un- 
dergone PCNL for stone disease in our department between March 2004 and October 2011. Eight patients had a previous contralateral nephrectomy (44.4\%), 4 patients had an anatomy solitary kidney (23.3\%), and 6 patients had nonfunctional contralateral kidneys (33.3\%). Non-functional contralateral kidneys were confirmed by nephro-dynamic imaging. Twelve patients underwent PCNL in prone position. Six patients underwent PCNL in completely supine position. Patient demographic characteristics, including gender, age, body mass index (BMI), history of shock wave lithotripsy (SWL), mean maximum stone diameter, hydronephrosis and previous kidney surgery (open and/or PCNL) were recorded. All surgeries were performed by the same surgeon. The operation style was decided after the common discussion of the surgeon and the patient. Informed consent was obtained from patients before operation. The study protocol was approved by Institutional Review Board of the First Hospital of Jilin University.

Preoperatively, widespectrum antibiotics were administered to patients with bacteriuria by experience (Cefuroxime Sodium or Ciprofloxacin Lactate), or patients were treated according to the antibiogram results. Operation could be done if the results of urinalysis and urine culture follow up testing became negative. Serum creatinine (Cr), systolic blood pressure, diastolic blood pressure, and new onset hypertension were determined preoperatively and postoperatively at 3 months.

\section{Equipment and instruments}

18-gauge coaxial needle (COOK Inc.), Zebra guide wire(Boston Scientific Corporation), fascial dilators(COOK Inc.), X-Force N30 Nephrostomy Balloon Dilation Catheter (BCR Inc.), F9 Olympus ureteroscope (Germany), F20 Storz nephroscope (Germany), Cybersonics Double-catheter system (America), Lumenis 60w holmium lithotripter (USA), Fluoroscopic table (Siemens), Aloka 5 multicolor ultrasound instrument with transducer frequency $3.5 \mathrm{MHz}$.

\section{Technique of PCNL}

The entire procedure was performed on the fluoroscopic table with the patient under general anesthesia.

\section{Prone position (Group A)}

After placing the patient in lithotomic position, retrograde ureter catheterization with a 5-French open-ended ureter catheter was performed. All other procedures were completed in the prone position. Under the guide of ultrasound, the coaxial needle was placed in the desired calyx. The working channel was then dilated by using the plastic dilator system or X-Force Nephrostomy Balloon Dilation Catheter to either F18 or F26. And then, the F9 ureteroscope or the F20 nephroscope was placed directly into the kidney through the established tract. The Lumenis 60w lithothiptor or Cybersonics Double-catheter system was used to fragment the renal stone.

\section{Completely supine position (Group B)}

The patients were placed in a completely supine position with the flank to be operated raised and slightly rotated by a single underlying 3-liter water bag. The procedure was the same to prone position.

Stone clearance was determined by a combination of fluoroscopy and ultrasound. At the end of the procedure, a double $J$ tube was placed within the ureter. And a clamped $14 \mathrm{~F}$ or $20 \mathrm{~F}$ Foley catheter was placed as a nephrostomy tube and it was opened within 24 hours. We rechecked KUB or ultrasound 1 or 2 days post-operation. And the nephrostomy tube was removed if there was no extravasation and larger residual stones at approximately 3 days post-operation. We routinely removed the double $\mathrm{J}$ tube about 1 month post-operation in the Outpatient Clinic.

Patients were considered stone-free when no stone $>4 \mathrm{~mm}$ was visualized. Residual fragments $>5 \mathrm{~mm}$ in diameter were treated with extracorporeal shock wave lithotripsy (ESWL) or second phase PCNL. Both of them were performed 1 week postoperatively.

\section{Statistical analysis}

Comparisons between continuous variables were performed using the Student $t$ test while comparisons between categorical variables were performed using a Pearson chi-square test (SPSS 13.0, SPSS Inc, Chicago, IL). $\mathrm{P}<0.05$ was considered to be statistically significant. 


\section{RESULTS}

The patients in group A were equally matched to those in group $\mathrm{B}$ cohort with regards to sex ratio, age, body mass index (BMI), stone location, stone size, existence of hydronephrosis, previous open surgery and ESWL ( $p>0.05)$ (Table-1). The main intraoperative and postoperative parameters are summarized in Table-2. The mean operative time was 104 minutes (range: 72-145 minutes) and 128 minutes (range: 80-170 minutes), respectively $(p>0.05)$. The I stage stone free rate was $91.7 \%$ and $83.3 \%$, respectively ( $p>0.05)$. One patient in group A who had residual fragments required drug therapy. While another patient in group B who had residual fragments underwent ESWL. No blood transfusions were required and no abdominal or thoracic organ injuries were reported in both groups.

There was no new onset hypertension by the end of follow-up in both groups (Table-3). Blood pressure levels in group A were equally matched to those in group B with regard to pre-operation and 3 months after operation. Systolic blood pressure showed a statistically significant improvement in group B ( $\mathrm{p}=0.034)$, but a non-statistically significant improvement in group A ( $p=0.368$ ) by the end of follow-up period. There were no statistical improvement in both groups about diastolic blood pressure before and 3 months after operation ( $p=0.275$ and 0.363 , respectively). Baseline values of serum creatinine were comparable in the two patients groups $(\mathrm{P}=0.92$ before operation and $\mathrm{P}=0.783$ by the end of follow-up). Both groups showed a similar fall in serum creatinine at 3 month follow-up period ( $\mathrm{p}=$ 0.004 and 0.029, respectively).

\section{DISCUSSION}

"Correct position, half operation" is one of the dogmas in surgery. So does in PCNL. The correct position of the patient during PCNL has always been a debated issue, as the precise access to the kidney is facilitated by a careful positioning of the patient and can reduce intraoperative complications (3-5).

PCNL is traditionally performed in the prone position for a safe approach to the kidney.

Table 1 - Patient characteristics.

\begin{tabular}{|c|c|c|c|}
\hline & $\begin{array}{l}\text { Group A } \\
\text { (prone) }\end{array}$ & $\begin{array}{c}\text { Group B } \\
\text { (completely supine) }\end{array}$ & $P$ value \\
\hline Total patients & 12 & 6 & \\
\hline $\mathrm{M} / \mathrm{F}$ ratio & $8: 4$ & $4: 2$ & 1.0 \\
\hline Mean age, yr(range) & $43.8(30-54)$ & $44.8(29-52)$ & 0.881 \\
\hline Mean BMI, kg/m² & $24.2(18.6-28.7)$ & $24.5(19.0-28.5)$ & 0.804 \\
\hline Side,right/left, n & $7: 5$ & $3: 3$ & 0.737 \\
\hline Mean maximum stone diameter,cm(range) & $3.3(2.3-4.8)$ & $3.6(2.2-5.0)$ & 0.568 \\
\hline Hydronephrosis yes/no & $9: 3$ & $4: 2$ & 0.710 \\
\hline Previous open surgery (\%) & $3(25)$ & $1(16.7)$ & 0.688 \\
\hline History ESWL (\%) & $10(83.3)$ & $5(83.3)$ & 1.0 \\
\hline
\end{tabular}


Table 2 - Intraoperative and postoperative parameters.

\begin{tabular}{lccc}
\hline & Group A (prone) & $\begin{array}{c}\text { Group B } \\
\text { (completely supine) }\end{array}$ & P value \\
\hline No. of access tracts & 10 & 6 & 0.289 \\
Simple & 2 & 0 & 0.077 \\
Complex & $104(72-145)$ & $128(80-170)$ & 0.596 \\
Mean operating time, min(range) & $91.7(11 / 12)$ & $83.3(5 / 6)$ & 0.488 \\
Stone free rate, \% & $-2.06(3.5-0.5)$ & $-2.12(3.6-0.5)$ & 0.18 \\
Mean blood loss $(\triangle \mathrm{Hb}), \mathrm{g} / \mathrm{dL}$ & $4(33.3)$ & 0 & 0.43 \\
Postoperative fever, $\mathrm{n}(\%)$ & $9.08(6-11)$ & $9.17(6-12)$ & 0.146 \\
Mean hospital stay, d(range) & 0 & 1 & 0 \\
II stage ESWL, $\mathrm{n}(\%)$ & 0 & 0 & \\
II stage PCNL, $\mathrm{n}(\%)$ & 0 & 0.48 \\
Major complications, no. of patients & 0 & \\
\hline
\end{tabular}

Major complications included septicemia, hemorrhage requiring blood transfusion, thoracic or abdominal organ injury, acute pancreatitis.

The prone position has some inherent merits (6-8). For example, a wide surgical field for the selection of the puncture site, an adequate nephroscopic manipulation, and a good distention of the collecting system. However, the prone position is often associated with a limitation in respiratory movements and potential anesthesia danger.

Recently, there have been many reports about PCNL in the supine position (9-11). The potential advantages of supine position in PCNL are as follows. Firstly, the surgeon can work while sitting on chairs during the whole procedures which is more comfortable. Secondly, the supine position has important anesthesiological advantages, such as a low incidence of cardiovascular and respiratory problems. Some authors believe that the incidence of colonic injuries is lower in supine position than that in prone position $(11,12)$. What's more, the supine position allows a simultaneous retrograde approach to the ureter and renal pelvis, with both rigid and flexible scopes, for contemporaneous treatment of ureteral or complex renal stones. However, every coin has its two sides, so does the supine position. The obviously antero- medial movement of the kidney during dilation makes the procedure more difficult. Besides, the superior calyceal puncture is more challenging. A narrow surgical field for the selection of the puncture site is another shortcoming of the supine position.

Valdivia Uria et al. (12) proposed PCNL in a completely supine position with a 3 liter water bag below the ipsilateral flank and with the ipsilateral leg totally extended. In their report on 557 patients, the procedure was successful in 93\% of the cases, with a low complication rate and no colonic perforation. We adopted this position in our department.

Treatment of staghorn stones in patients with solitary kidney is one of the most difficulties in PCNL (13). Major complications, although rare, can lead to significant morbidity especially in patients with solitary kidney. This study shows it is safe both in prone position and modified position. No blood transfusions were required and no abdominal or thoracic organ injuries were reported in both groups. The stone free rate of solitary kidney PCNL in group A and group B was 91.7\% and 83.3\%, res- 
Table 3 - Blood pressure levels and serum creatinine before and after PCNL.

\begin{tabular}{lccc}
\hline & Group A (prone) & Group B (completely supine) & P value \\
\hline New onset hypertension & 0 & 0 & \\
Systolic blood pressure (mmHg) & & & \\
$\quad$ Preoperative & $127.5 \pm 14.5$ & $132.5 \pm 14.1$ & 0.8 \\
$\quad$ Postoperative, 3 months & $125.8 \pm 10.8$ & $126.7 \pm 11.7$ & 0.97 \\
Improvement of blood pressure & $1.37 \pm 6.15$ & & 0.368 \\
Improvement of blood pressure & & $5.83 \pm 4.92$ & 0.034 \\
Diastolic blood pressure (mmHg) & & & 0.283 \\
Preoperative & $80 \pm 8.53$ & $82.5 \pm 11.7$ & 0.167 \\
Postoperative, 3 months & $78.8 \pm 6.4$ & $80.8 \pm 9.7$ & 0.275 \\
Improvement of blood pressure & $1.25 \pm 3.77$ & & 0.363 \\
Improvement of blood pressure & & $1.67 \pm 4.08$ & 0.029 \\
Serum creatinine (mg/dL) & & & 0.92 \\
Preoperative & $1.18 \pm 0.31$ & $1.24 \pm 0.32$ & 0.783 \\
Postoperative, 3 months & $1.00 \pm 0.17$ & $0.98 \pm 0.13$ & $0.04 \pm 4.92$ \\
Improvement of blood serum creatinine & $1.37 \pm 6.15$ & & \\
Improvement of blood serum creatinine & & & \\
\hline
\end{tabular}

pectively. The stone free rate was higher in group A than that in group B, but this was not statistically significant $(p=0.596)$. Four patients $(33.3 \%)$ in the group A had postoperative fever. This was in contrast to the group B, of which none of the patients had postoperative fever. The probable reason is that the percutaneous tract is horizontal or slightly inclined downward, spontaneous evacuation of stone fragments during the procedure is easier and the pressure inside the pelvis is low in supine position.

Study of blood pressure in patients with solitary kidney and staghron stonesis few. Berkan et al. (14) observed staghorn stones in 16 patients. The number of patients with hypertension before PCNL was five and by the end of follow-up there was no new onset hypertension. The result is similar with us. We also observed that systolic blood pressure showed a statistically significant improvement in group B. The reason why systolic blood pressure improved after 3 months instead of diastolic blood pressure was unclear.

A few studies have investigated the factors that affect renal function in patients with solitary kidney (15-19). Most of them demonstrated a significant improvement in creatinine or GFR levels from preoperatory levels to about 1-year of follow-up. In this study, we observed that both groups showed a similar fall in serum creatinine at 3 month follow-up period $(\mathrm{p}=0.004$ and 0.029 , respectively).

However, the number of cases in the study was comparatively small, which result in lack of enough confidence on statistical analysis of the data. The reasons of fewer patients are a short 
study period and a comparatively lower incidence rate of staghorn stones in patients with solitary kidney. However, we believe that our research will give some inspiration to new studies that should also estimate the preoperative and postoperative GFR and compare them.

\section{CONCLUSIONS}

PCNL is safe and has an acceptably high stone free rate in patients with solitary kidneys. At short-term follow-up, systolic blood pressure has improved in PCNL in supine position.

\section{CONFLICT OF INTEREST}

None declared.

\section{REFERENCES}

1. Yuan H, Zheng S, Liu L, Han P, Wang J, Wei Q: The efficacy and safety of tubeless percutaneous nephrolithotomy: a systematic review and meta-analysis. Urol Res. 2011; 39: 401-10.

2. Akman T, Binbay M, Tekinarslan E, Ozkuvanci U, Kezer C, Erbin A, et al.: Outcomes of percutaneous nephrolithotomy in patients with solitary kidneys: a single-center experience. Urology. 2011; 78: 272-6.

3. Falahatkar S, Allahkhah A, Soltanipour S: Supine percutaneous nephrolithotomy: pro. Urol J. 2011; 8: 257-64.

4. Valdivia JG, Scarpa RM, Duvdevani M, Gross AJ, Nadler RB, Nutahara K, et al.: Supine versus prone position during percutaneous nephrolithotomy: a report from the clinical research office of the endourological society percutaneous nephrolithotomy global study. J Endourol. 2011; 25: 1619-25.

5. Liu L, Zheng S, Xu Y, Wei Q: Systematic review and metaanalysis of percutaneous nephrolithotomy for patients in the supine versus prone position. J Endourol. 2010; 24: 1941-6.

6. Cracco CM, Scoffone CM: ECIRS (Endoscopic Combined Intrarenal Surgery) in the Galdakao-modified supine Valdivia position: a new life for percutaneous surgery? World J Urol. 2011; 29: 821-7.
7. Duty B, Waingankar N, Okhunov Z, Ben Levi E, Smith A Okeke Z: Anatomical variation between the prone, supine, and supine oblique positions on computed tomography: implications for percutaneous nephrolithotomy access. Urology. 2012; 79: 67-71.

8. Wang $\mathrm{Y}$, Jiang $\mathrm{F}$, Wang $\mathrm{Y}$, Hou $\mathrm{Y}$, Zhang $\mathrm{H}$, Chen, et al.: Post-percutaneous nephrolithotomy septic shock and severe hemorrhage: a study of risk factors. Urol Int. 2012; 88: 307-10.

9. Hoznek A, Rode J, Ouzaid I, Faraj B, Kimuli M, de la Taille A, et al.: Modified supine percutaneous nephrolithotomy for large kidney and ureteral stones: technique and results. Eur Urol. 2012; 61: 164-70.

10. Falahatkar S, Asli MM, Emadi SA, Enshaei A, Pourhadi H, Allahkhah A: 10 Complete supine percutaneous nephrolithotomy (CSPCNL) in patients with and without a history of stone surgery: safety and effectiveness of CSPCNL. Urol Res. 2011; 39: 295-301.

11. Falahatkar S, Farzan A, Allahkhah A: Is complete supine percutaneous nephrolithotripsy feasible in all patients? Urol Res. 2011; 39: 99-104.

12. Valdivia Uría JG, Lachares Santamaría E, Villarroya Rodríguez S, Taberner Llop J, Abril Baquero G, Aranda Lassa JM. Percutaneous nephrolithectomy: simplified technic (preliminary report). Arch Esp Urol. 1987; 40: 177-80.

13. Osther PJ, Razvi H, Liatsikos E, Averch T, Crisci A, Garcia $\mathrm{JL}$, et al.: Percutaneous nephrolithotomy among patients with renal anomalies: patient characteristics and outcomes; a subgroup analysis of the clinical research office of the endourological society global percutaneous nephrolithotomy study. J Endourol. 2011; 25: 1627-32.

14. Resorlu B, Kara C, Oguz U, Bayindir M, Unsal A: Percutaneous nephrolithotomy for complex caliceal and staghorn stones in patients with solitary kidney. Urol Res. 2011; 39: 171-6.

15. Akman T, Binbay M, Aslan R, Yuruk E, Ozgor F, Tekinarslan $E$, et al:: Long-term outcomes of percutaneous nephrolithotomy in 177 patients with chronic kidney disease: a single center experience. J Urol. 2012; 187: 173-7.

16. Basiri A, Shabaninia S, Mir A, Soltani MH: The safety and efficacy of percutaneous nephrolithotomy for management of large renal stones in single- versus doublefunctioning kidney patients. J Endourol. 2012; 26: 235-8.

17. Bucuras V, Gopalakrishnam G, Wolf JS Jr, Sun Y, Bianchi G, Erdogru T, et al.: The Clinical Research Office of the Endourological Society Percutaneous Nephrolithotomy Global Study: nephrolithotomy in 189 patients with solitary kidneys. J Endourol. 2012; 26: 336-41. 
18. Bayram A, Esmaoglu A, Akin A, Baskol G, Aksu R, Bicer C, et al.: The effects of intraoperative infusion of dexmedetomidine on early renal function after percutaneous nephrolithotomy. Acta Anaesthesiol Scand. 2011; 55: 539-44.
19. Akman T, Sari E, Binbay M, Yuruk E, Tepeler A, Kaba M, et al.: Comparison of outcomes after percutaneous nephrolithotomy of staghorn calculi in those with single and multiple accesses. J Endourol. 2010; 24: 955-60.

Correspondence address:

Dr. Chunxi Wang

Department of Urology

The First Hospital of Jilin University

71 Xinmin ST

Changchun, Jilin Province

Telephone: +04 318878-2321

E-mail: chunxi_wang@126.com 